\title{
Real-time ultrasound-guided external intracerebral hemorrhage drain placement
}

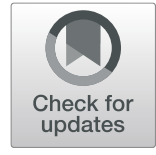

\author{
Cong Feng ${ }^{1 \dagger} \mathbb{D}$, Sai Huang ${ }^{2 \dagger}$, Li Chen ${ }^{1 \dagger}$, Xuan Zhou', Li-Li Wang ${ }^{1}$, Fa-Qin Lv ${ }^{3 *}$ and Tan-Shi Li ${ }^{1 *}$
}

\begin{abstract}
We report a new minimally invasive technique utilizing interventional ultrasound for precise external intracerebral hemorrhage drain (EICHD) placement in pigs.
\end{abstract}

Keywords: Ultrasound guidance, Intracerebral hemorrhage, External drain

\section{Background}

The severity of cerebral injury is related to the development and formation of a hematoma, which could cause secondary events, including elevated intracranial pressure (ICP) and edema. It is critical that large hematomas are evacuated at an early stage. Catheter drainage-based techniques are notable examples of minimally invasive techniques for intracerebral hemorrhage ( $\mathrm{ICH}$ ) evacuation [1]. These catheter insertion approaches are assisted by many kinds of imaging techniques and can be performed through a small burr hole. However, many procedures cannot be performed at the bedside because they usually require surgical neuronavigation or the assistance of other large imaging equipment. Here, we described a minimally invasive interventional technique using real-time ultrasound guidance through a small burr hole to achieve external intracerebral hemorrhage drainage (EICHD) placement.

\section{Methods}

Three healthy male miniature pigs weighing $10 \pm 1 \mathrm{~kg}$ were anesthetized by intramuscular injection of $3 \%$

\footnotetext{
* Correspondence: Ivjin8912@163.com; Its301@163.com

${ }^{+}$Cong Feng, Sai Huang and Li Chen contributed equally to this work as cofirst authors.

${ }^{3}$ Department of Ultrasound, Hainan Hospital, Chinese PLA General Hospital, Sanya 572000, China

'Department of Emergency, the First Medical Center of Chinese PLA General Hospital, Beijing 100853, China

Full list of author information is available at the end of the article
}

pentobarbital sodium $(30 \mathrm{mg} / \mathrm{kg})$. We mimicked the clinical conditions of traumatic ICH using an animal model of parenchymal hematoma that was established by stereotactic injection of $3 \mathrm{~mL}$ of nonanticoagulated autologous arterial blood into the right lobe of the brain parenchyma $20 \mathrm{~mm}$ from the skull [2].

After the model was established, each brain was subjected to a transcranial ultrasound examination (Bmode and color Doppler) at the back of the ear to identify the parenchymal hematoma (Fig. 1a). After identification of the parenchymal hematoma, the ultrasound probe was adjusted so that the puncture guideline could pass through the center of the parenchymal hematoma, and the exact entry point of the EICHD could also be determined (Additional file 1: Figure S1). The distance from the guide channel entrance to the center of hematoma could be calculated (Fig. 1b). Then, to accommodate the 16-gauge needle (outer diameter $1.6 \mathrm{~mm}$ ) and catheter (outer diameter $3.0 \mathrm{~mm}$ ), a $7.0-\mathrm{mm}$-diameter burr hole was made at the determined entry point using a $7-\mathrm{mm}$ perforator drill under the guidance of the ultrasound. After burr hole establishment, we performed an EICHD insertion under real-time ultrasound guidance (Fig. 1c).

\section{Results}

After the catheter was placed slightly in the parenchymal hematoma (Fig. 1d) and blood could be seen flowing out of the catheter (Additional file 1: Figure S2), the process 

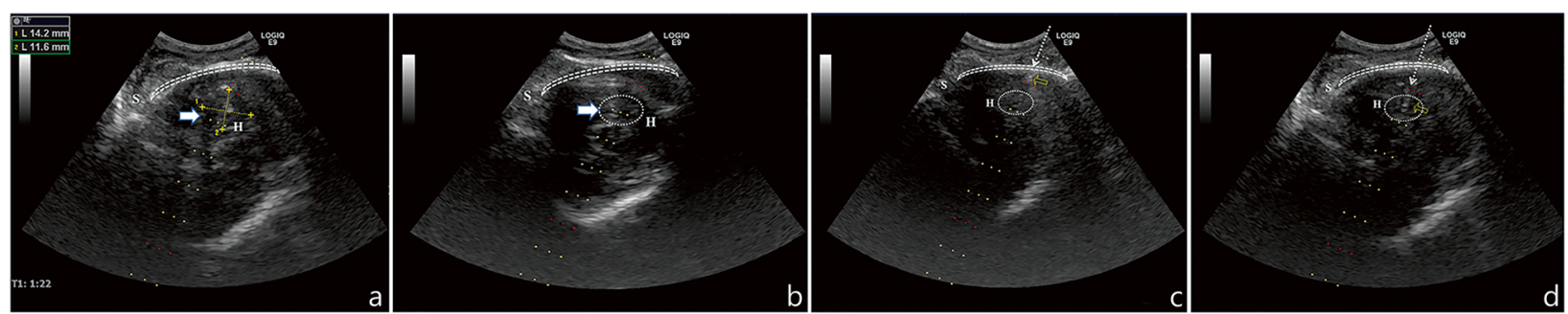

Fig. 1 Operative process of ultrasound images showing the target intracerebral hematoma. a: The parenchymal hematoma was formed by autologous blood injection (as shown by the white arrow), and the longest diameter is approximately $14.2 \mathrm{~mm}$ (as shown by the yellow dotted line 1). b: The parenchymal hematoma was identified (as shown by the white arrow and white dotted circle). The ultrasound probe was adjusted so that the center of the puncture guideline passed through the parenchymal hematoma. We know that the distance from the guide channel entrance to the center of hematoma (dot on the image center puncture guideline) is approximately $30 \mathrm{~mm}$. c: Real-time tracking of the EICHD following the dotted puncture guideline as indicated by the white dotted arrow. The catheter passed through the burr hole in the skull under real-time ultrasound guidance, and the catheter position is indicated by the yellow arrow. $\mathbf{d}$ : The catheter reached the correct position in the hematoma. (The catheter is indicated by the white dotted arrow, and the final position is indicated by the yellow arrow). H: Hematoma, S: Skull as shown by the white dotted circle

of EICHD was classified as successful, and the catheter could be positioned and secured well.

\section{Discussion}

Traumatic cerebral injury usually leads to intracerebral hematoma with a mass effect, and EICHD could aid in decompression, which might help to reduce the mortality and disability rate. Recently, with the development of ultrasound equipment, some studies have reported that ultrasound-guided catheter drainage in clinical ICH cases could achieve EVD placement through a burr hole [3]. Therefore, we attempted a new minimally invasive technique of real-time ultrasound-guided EICHD placement with commonly used ultrasonic and other interventional equipment that could be operated at the bedside and resulted in much less invasive damage to the patient.

We have tested a new minimally invasive approach of local hemostatic drug therapy by the real-time guidance of transcranial contrast-enhanced ultrasound (CEUS) based on the same model of traumatic ICH [4]. This study is a continuation and extension of our previous study and forms a set of effective treatment methods for $\mathrm{ICH}$.

However, this new technique also has many limitations. First, because the physiologic window of the skull was limited and the window was relatively narrow, hematomas in all areas of the brain could not be scanned using the current method. Second, this minimally invasive technique could be performed only if the hematoma had been clearly identified. Third, the detection of the hematoma location by transcranial ultrasound was limited due to the two-dimensional images and the limited physiologic window of the skull. Therefore, this technique still requires further investigation.

\section{Supplementary information}

Supplementary information accompanies this paper at https://doi.org/10 1186/s40779-020-00261-9.

Additional file 1: Figure S1. One interventional burr hole is placed for the EICHD catheter, whose positions are adjusted by the ultrasound transducer. Figure S2. Blood could be seen flowing out of the catheter.

\section{Abbreviations}

EICHD: External intracerebral hemorrhage drain; ICH: Intracerebral hemorrhage; ICP: Intracranial pressure

\section{Authors' contributions}

TSL, FQL and LC designed the study. CF and XZ performed experiments. SH and LLW helped with data collection and statistical analysis. All authors read and approved the final manuscript.

\section{Funding}

The present study was supported by grants from the Beijing Science and Technology New Star Project (XX2018019/Z181100006218028), the National Natural Science Fund (81701961), the PLA Logistic Major Science and Technology Project (16QNP135), the PLA General Hospital Science and Technology Project (2018XXFC-20, 16KMM56, 2017FC-WJFWZX-30), the Winter Olympics of Science and Technology (2019YFF0302300), and the Construction Project of Key Disciplines in the 13th Five-Year Plan of the PLA (Traumatic Surgery in the Battlefield, 2019-126, 2019-513).

Availability of data and materials Not applicable.

\section{Ethics approval and consent to participate}

The experimental protocol was approved by the Ethics Committee for Animal Research from the General Hospital of the People's Liberation Army (PLA), and all experimental pigs received humane care.

Consent for publication

Not applicable. 


\section{Competing interests}

The authors declare that they have no competing interests.

\section{Author details}

'Department of Emergency, the First Medical Center of Chinese PLA General Hospital, Beijing 100853, China. ${ }^{2}$ Department of Hematology, the First

Medical Center of Chinese PLA General Hospital, Beijing 100853, China.

${ }^{3}$ Department of Ultrasound, Hainan Hospital, Chinese PLA General Hospital,

Sanya 572000, China.

Received: 11 September 2019 Accepted: 11 June 2020

Published online: 02 July 2020

\section{References}

1. Mould WA, Carhuapoma JR, Muschelli J, Lane K, Morgan TC, McBee NA, et al. Minimally invasive surgery plus recombinant tissue-type plasminogen activator for intracerebral hemorrhage evacuation decreases perihematomal edema. Stroke. 2013;44(3):627-34.

2. Bullock R, Mendelow AD, Teasdale GM, Graham DI. Intracranial haemorrhage induced at arterial pressure in the rat. Part 1: description of technique, ICP changes and neuropathological findings. Neurol Res. 1984; 6(4):184-8.

3. Manfield $\mathrm{JH}, \mathrm{Yu} \mathrm{KKH}$. Real-time ultrasound-guided external ventricular drain placement: technical note. Neurosurg Focus. 2017;43(5):E5.

4. Feng C, Huang S, Zhou X, Wang L, Cui X, Chen L, et al. Interventional ultrasound assisted early local hemostatic drug therapy in traumatic intracerebral hemorrhage. Ultrasound Med Biol. 2020;46(1):180-7.

Ready to submit your research? Choose BMC and benefit from:

- fast, convenient online submission

- thorough peer review by experienced researchers in your field

- rapid publication on acceptance

- support for research data, including large and complex data types

- gold Open Access which fosters wider collaboration and increased citations

- maximum visibility for your research: over $100 \mathrm{M}$ website views per year

At $\mathrm{BMC}$, research is always in progress.

Learn more biomedcentral.com/submissions 